\title{
CONTRIBUCIONES ESPECIALES AL DEBATE
}

\author{
Alejandro Moreno ${ }^{1}$ y Elizabeth J. Zechmeister
}

\section{Alejandro Moreno}

Medir la opinión pública a través de encuestas es una labor que, desde sus orígenes, los encuestadores hemos visto como una aportación a la vida democrática. Sin embargo, como en otros momentos de su historia, las encuestas están hoy bajo sospecha.

Hacer un listado de las aportaciones mutuas de encuestas y democracia sería muy ambicioso y tomaría mucho tiempo, pero destaco algunas de las que se ven hoy en nuestros países. Primero, las encuestas registran la pluralidad de opiniones y voces ciudadanas sobre los temas de interés público. Ejemplo: cuatro de cada diez mexicanos se preocupan todo o casi todo el tiempo de que puedan llegar a ser víctimas de un delito con violencia. ${ }^{2}$

Segundo, las encuestas reportan los sentimientos de la gente respecto de la labor de los gobiernos y las instituciones. En el segundo trimestre de este 2016, la proporción de ciudadanos que dijo aprobar la labor del presidente era de tres cuartas partes en República Dominicana, poco más de la mitad en Bolivia, un tercio en Colombia, una cuarta parte en México y una quinta parte en Brasil, Venezuela y Perú. ${ }^{3}$ A su vez, en esos mismos países la proporción de ciudadanos que opina que la democracia es el mejor sistema de go-

\footnotetext{
${ }^{1}$ Doctor en Ciencia Política por la Universidad de Michigan, profesor de Ciencia Política en el ITAM, ex presidente de la World Association for Public Opinion Research (Wapor), vicepresidente de la World Values Survey Association (WVSA), consultor y director de encuestas de opinión pública, El Financiero.

${ }^{2}$ Fuente: Latinobarómetro 2016.

3 Ídem.
} 
bierno varía entre 70 y 85 \%. Son sociedades que apoyan a la democracia pero son críticas hacia sus gobiernos.

Tercero, las encuestas muestran la distribución de preferencias y posturas de los grupos sociales ante propuestas de legislación y política pública. Ante la iniciativa que el presidente de México envió al Congreso para legalizar el matrimonio igualitario -o entre personas del mismo sexo-, el $37 \%$ de los mexicanos dijo estar de acuerdo con la medida y $55 \%$ en desacuerdo. No obstante, entre los jóvenes menores de 30 años se registró un apoyo del 61\% a la medida, mientras que entre los mayores de 50 años el rechazo alcanzó el $80 \%$.

Cuarto, las encuestas captan las reacciones públicas a los mensajes, acciones y conducta de los líderes políticos. En la misma temática del matrimonio igualitario, el gobernador de Nuevo León hizo declaraciones en las que daba a entender que el matrimonio de personas del mismo sexo no es bienvenido en el estado y les pedía que se fueran a otro. Una encuesta realizada en esa entidad mostró que el 19\% de los entrevistados consideró adecuadas las declaraciones del gobernador, mientras que el $61 \%$ las calificó como inadecuadas. ${ }^{5}$

Quinto, en una de sus facetas más protagónicas, las encuestas miden puntualmente el apoyo o rechazo a las opciones que compiten en los procesos electorales; asisten la información periodística y el análisis durante las campañas $\mathrm{y}$, de manera muy importante, guían las decisiones de los partidos políticos para definir candidaturas, plantear propuestas y mensajes y detectar fortalezas y debilidades en su intento por ganar apoyo popular en las urnas. A casi dos años de distancia, las encuestas sobre intención de voto ya delinean escenarios de competencia para la elección presidencial de 2018 en México. Las encuestas nos permiten tener una buena idea de la distribución de preferencias a nivel nacional, pero también adentrarnos a la manera en la que ciertos subgrupos opinan y se comportan. Por ejemplo,

${ }^{4}$ Encuesta nacional El Financiero-Alejandro Moreno, 1 julio 2016.

${ }^{5}$ Encuesta estatal en Nuevo León, El Financiero-Alejandro Moreno, 17 octubre 2016. 
la tercera parte de mexicanos poseedores de teléfono inteligente expresa preferencias políticas muy distintas a las de los mexicanos que no poseen teléfono inteligente. Esas diferencias, lo que en otro panel de este congreso denomino el smartphone cleavage, tienen implicaciones fundamentales para las nuevas formas de hacer campañas, para ganar el voto de la generación Milenial-Facebook.

Las bases técnicas, científicas, probabilísticas y replicables de las encuestas les han provisto de legitimidad para documentar, con un alto grado de confiabilidad, los estados de ánimo de la opinión pública. Las encuestas pesan políticamente no porque sean un fin en sí, sino por ser un medio por el cual la opinión pública se articula y se vuelve efectiva en la arena política, para tomar prestado un término de Philip Converse. ${ }^{6}$ Si las encuestas aportan a la democracia es porque han sido capaces de dar forma, sentido y significado a la opinión pública y a sus diversos subgrupos. Como bien sabemos, la opinión pública es un factor vital de la democracia.

Sin embargo, las encuestas se han puesto una vez más bajo sospecha. Se dice que, más que contribuir a la vida democrática, como alguna vez lo hicieron, hoy le resultan dañinas. Algunos críticos han sentado a las encuestas en el banquillo de los acusados. Testifican en su contra, denuncian sus fallas, señalan sus sesgos, subrayan los intereses que las mueven y, en los casos más sofisticados, argumentan que las metodologías ya son inadecuadas y no logran captar a las sociedades cambiantes de hoy. Algunos pregonan que las encuestas están en una fase terminal, por lo que no es conveniente pedirles que sigan asistiendo a las necesidades de información que la democracia requiere.

Las encuestas de opinión pública, y sobre todo las electorales, tienen un historial de amor y odio con el público informado que las consume, que las reporta y que las discute. Las encuestas, fuentes primordiales de información, recursos

\footnotetext{
${ }^{6}$ Ver Philip E. Converse, "Changing Conceptions of Public Opinion in the Political Process". Public Opinion Quarterly, vol. 51: S12-S24.
} 
imprescindibles de la vida electoral, contenidos de primera plana de los diarios, se respetan hasta que "se equivocan". Entonces se vuelven blanco de ataque y objeto de desdén. Si aciertan, cumplieron; si fallan, las embiste la crónica de una muerte anunciada. Pero no sin antes condenarlas. La sentencia de culpabilidad procede por lo regular sin un juicio justo y ante un jurado pasional. En esta era de Facebook, Twitter y de otras redes sociales, el menosprecio por las encuestas puede ser viral.

¿Qué hacer? Un foro como este, el Séptimo Congreso Latinoamericano de Wapor (Asociación Mundial de Investigadores de Opinión Pública), es el sitio ideal para hacer una profunda reflexión acerca de la labor que llevamos a cabo los encuestadores en los diversos países de la región. Durante estos dias discutiremos encuestas, sus resultados y metodologías (una mirada rápida al programa muestra temas de innovación metodológica, cambios sociales, realineaciones políticas, evaluaciones de políticas públicas, uso de medios tradicionales y de nuevas tecnologías de información, la conducta de los votantes, la participación ciudadana, la confianza y la desconfianza en las instituciones, entre otros) y plantearemos viejas preguntas con nuevas respuestas y nuevas preguntas en busca de respuesta. Mientras escuchamos las presentaciones de nuestros colegas, reflexionemos: iestamos aportando a la democracia con nuestras encuestas en nuestras respectivas sociedades? ¿o estamos dañando la salud democrática con nuestras estimaciones y sus distintas interpretaciones?

En un artículo publicado en la revista The New Yorker, de noviembre de 2015, la historiadora Jill Lepore lanzó la siguiente acusación: las encuestas están arruinando a la democracia. Cito: "Las encuestas nunca han sido más influyentes y nunca han sido menos confiables que ahora". Como si tratara de reproducir las aventuras del joven Haroun en las novelas de Salman Rushdie, ${ }^{7}$ Lepore sugiere que, en

${ }^{7}$ Salman Rushdie, Haroun and the Sea of Stories. 
su ruta a una elección, los candidatos deben atravesar un "mar de encuestas", un mar que hoy es más profundo y más oscuro que nunca, según afirma la escritora. Donald Trump, dice Lepore, es una criatura de ese mar. La metáfora deja muy clara la impresión de que el mar de encuestas es peligroso: el navegante enfrenta crecientes olas de rechazo y torrentes de no cooperación que debilitan la representatividad y la solidez de los navíos muestrales. Esto afecta los instrumentos y, por supuesto, los mapas de navegación: los resultados no reflejan un universo completo, solamente uno observable bajo condiciones adversas. Las filtraciones de votantes probables, como las de agua, añaden riesgos, ya que, si bien sirven para mejorar los pronósticos electorales, anulan a porciones importantes de la opinión pública que deciden no expresarse en las urnas. Las encuestas van con la brújula descompuesta, con la vela rota y, en el peor de los casos, con navegantes poco habilidosos para enfrentar aguas desconocidas.

Lepore señala el fracaso de las nuevas prácticas metodológicas que combinan modalidades de entrevista, telefonía móvil e Internet. Sobre todo, la crítica generalizada pone énfasis en las marcadas fallas para prever o pronosticar los resultados electorales. Pero el problema más grande de todos, dice Lepore, no es ni metodológico ni tecnológico es político. Cito: "Los encuestadores se volvieron prominentes bajo el clamor de que medir a la opinión pública es bueno para la democracia. Pero, ¿y si es malo?"

Aunque las críticas de Lepore se dirigen al contexto norteamericano, sus observaciones no son desdeñables para América Latina. De hecho, en México han tenido eco. Recientemente tuve un intercambio con un intelectual mexicano, a quien respeto profundamente, acerca de si las encuestas son dañinas para la democracia o no. ${ }^{8} \mathrm{~A}$ las encuestas hay que aplicarles una capa de escepticismo, ar-

${ }^{8}$ Intercambio de columnas periodísticas con Jesús Silva-Herzog Márquez, Reforma 8 y 15 de agosto de 2016; y El Financiero 12 y 19 de agosto de 2016. 
gumentaba Jesús Silva-Herzog Márquez en una columna periodística. Sí, hay que hacerlo, respondi, pero no como un gesto momentáneo sino como una actitud constante y, para que resulte más efectivo, hay que aplicarlo no solamente a la manera en que se realizan las encuestas, sino también a la manera en que se diseminan. La salud de las encuestas y su aportación a la democracia dependen no solamente de quiénes las hacen y de las metodologías que emplean; depende también de quiénes las difunden, las comentan y las interpretan. La cultura de encuestas incluye su hechura así como su lectura.

El citado intercambio periodístico tocó un segundo punto: admitir que las encuestas están en crisis. Mi postura es que sí, por supuesto, que hay crisis, pero no podemos quedarnos ahí: tenemos que discernir qué tipo de crisis es, cuándo comenzó y, lo más importante, cómo podemos salir de ella. En mi respuesta enumeré por lo menos cuatro tipos de crisis de las encuestas: una crisis de credibilidad, derivada de las fallas de estimación en diversas elecciones y plebiscitos; una crisis metodológica, en la que debemos preguntarnos si las encuestas deben adaptar sus metodologías o no a diversos factores de cambio, incluidas las tasas de cooperación, así como el cambio tecnológico, entre otros; una crisis de identidad, que refleja las dudas de diversos profesionales de la demoscopía acerca de cuál es su labor, particularmente en torno a la adecuada estimación de resultados electorales. Y una crisis existencial, en la que las encuestas comienzan a ser rebasadas por una nueva especie en la medición de opiniones y conductas, la nueva ciencia de datos, como la llama Lepore.

A simple vista, la crisis de credibilidad se atiende con un aceptable desempeño de las encuestas electorales. Pero no es tan sencillo. La regla con la que se mide el desempeño de las encuestas es asimétrica: la precisión y el acierto suelen ser discretos y se olvidan pronto; en contraste, la imprecisión y el error abren heridas que tardan en cicatrizar, en gran parte porque la crítica las sigue abriendo de vez en cuando. 
En su biografía de los hermanos Wilbur y Orville Wright, ${ }^{9}$ precursores de la aviación, David McCullough narra los distintos intentos de prueba y error que los hermanos llevaron a cabo una y otra vez para poner su nueva maquinaria en el aire de manera sostenida. En una de tantas pruebas, uno de los hermanos logró elevar su avión por unos momentos para luego caer de manera violenta al suelo. Disipado el polvo y los temores, el piloto repasó cada momento, cada acción, cada pieza mecánica y llegó a la siguiente conclusión: La aeronave funcionó bien, no falló ningún mecanismo, el clima era propicio, el viento favorable, las condiciones óptimas. Conclusión: el inventor-piloto se dio cuenta de que, para volar exitosamente, el aparato necesitaba un elemento adicional al buen funcionamiento mecánico y al clima favorable: la habilidad y pericia de quien va sentado en los controles. Máquina y habilidad humana eran, forzosamente, un dueto inseparable para enfrentar el viento en un clima favorable y, eventualmente, en uno menos favorable.

No dudo de que los encuestadores al mando de sus encuestas tienen la habilidad y la pericia, el entrenamiento y la voluntad de que sus estudios "vuelen" apropiadamente. Pero no está de más hacer una revisión integral; no sobra revisar metodologías, condiciones de vuelo y habilidades. El año pasado, las fallas de las encuestas en la elección para gobernador de Nuevo León, en México, fueron notables. Tuve oportunidad de hablar con encuestadores cuyas estimaciones erraron de manera significativa respecto del resultado de la elección y pocos sabían exactamente por qué. "Si tuviera que hacer todo de nuevo lo haría exactamente igual," me dijo uno de ellos. La profesión de encuestas se ha venido adaptando a nuevas circunstancias, a nuevos retos, $\mathrm{y}$, como muchos sabemos, a vientos cada vez más desfavorables. Es nuestra responsabilidad como investigadores reconocer la capacidad de vuelo de nuestras encuestas y hacer los ajustes necesarios, tanto al motor como al diseño, así

${ }^{9}$ David McCullough, The Wright Brothers, 2015. 
como a las propias habilidades de quien las pretende volar de manera sostenida.

Las encuestas aportan valiosamente a la democracia, pero es posible que, para desempeñarse a la altura, puedan necesitar una afinación, un buen mantenimiento $\mathrm{y}$, en algunos casos, un cambio de piezas. Su esencia es conocer la opinión pública por la vía de hacer preguntas a una muestra probabilística de la población de interés. Las formas, métodos, técnicas, logísticas, prácticas, costos y otros aspectos que las hacen posibles son diversos y cambiantes. Para quienes las hacemos, es fundamental tomarlos en cuenta. Para quienes las consumen, es muy importante reconocer sus tiempos, sus características y sus alcances.

Termino con una reflexión en torno a las habilidades para enfrentar las nuevas condiciones de vuelo en la medición de la opinión pública. El equipamiento de un piloto incluye el conocimiento de los instrumentos, sus habilidades y horas de vuelo, su capacidad y voluntad de innovación, su optimización de recursos humanos y presupuestales, pero también su apego a los principios éticos y de transparencia $\mathrm{y}$, sobre todo, su adhesión a un código de conducta responsable ante la sociedad, hacia los tomadores de decisiones y hacia la propia investigación de la opinión pública, con un sólido compromiso con la producción de datos con criterios de utilidad, precisión, exactitud, transparencia y replicabilidad. Con todo esto, creo que el vuelo de las encuestas aún tendrá muy buenos tiempos.

\section{Elizabeth J. Zechmeister ${ }^{10}$}

El escepticismo sobre la calidad y el rol de las encuestas de opinión pública, especialmente las encuestas electorales, no es nuevo (Asher, 2017). Recientes acontecimientos sobre sus aciertos y errores en países donde las encuestas de opi-

${ }^{10}$ La autora es Directora del Proyecto de Opinión Pública de América Latina (Lapop, por su sigla en inglés), profesora Cornelius Vanderbilt de Ciencia Politica en Vanderbilt University. Se la puede contactar en liz.zechmeister@vanderbilt.edu. 
nión son muy importantes han aumentado la prominencia de las dudas sobre ellas en el discurso público. Aunque es fácil dejarse llevar por el tono del debate del momento, esta nota presenta una opinión contraria que insta a adoptar una perspectiva diferente.

Me gustaría comenzar señalando que ha habido una explosión en la cantidad y avances importantes en la calidad de la investigación de encuestas alrededor del mundo, incluyendo América Latina. Dentro de la comunidad científica dedicada a este tipo de investigación, la tendencia general se encamina al uso de métodos más rigurosos, mayor transparencia y mayor escrutinio sobre cuestiones relacionadas con el diseño y la calidad de los datos. La disponibilidad de nuevas tecnologías y de innovaciones basadas en estos avances ha hecho posibles nuevos progresos y mejoras en los estudios sobre opinión pública y el comportamiento electoral. Uno de estos avances tiene que ver con la expansión de experimentos de encuestas, frecuentemente facilitados por la posibilidad de utilizar nuevas tecnologías. Algunos de los muchos frutos de esta labor colectiva aparecen en las páginas de revistas como Public Opinion Quarterly (POQ), International Journal of Public Opinion, Survey Research Methods y el Journal of Survey Statistics and Methodology. Mientras que la primera de estas revistas, POQ, data de la década de 1930, el resto tienen una más reciente creación que responde a la creciente preocupación de aspectos metodológicos de las encuestas. La segunda apareció en 1989, la tercera en 2007 y la cuarta en 2013. ${ }^{11}$ Existe cierta ironía en el aumento del escepticismo

\footnotetext{
${ }^{11}$ La creciente tendencia de publicaciones en revistas académicas de trabajos basados en métodos de investigación por muestreo y opinión pública en América Latina (Zechmeister y Seligson, 2012) también ha sido moldeada gracias al cumplimiento de las normas relativas al acceso de datos por académicos y firmas particulares, así como por proyectos de encuestas comparadas (entre países y/o en el tiempo) tales como el Barómetro de las Américas, el Latinobarómetro, el Mexico Panel Studies, Las Américas y el Mundo y otros de la forma adoptada por otras encuestas globales y regionales tales como el Comparative Study of Electoral Systems, el World Values Survey, el European Social Survey, el AfroBarometer, el Arab Barometer y el Asian Barometer, entre otros.
} 
hacia la investigación sobre opinión pública con encuestas justo cuando la disciplina ha llevado a cabo tan importantes avances.

Dicho esto, no hay duda de que el público mantiene una mirada escéptica sobre las encuestas. ¿Por qué? Como algunos han anotado en este simposio, este es en parte un problema político: algunas de las relaciones entre firmas encuestadoras y agentes políticos alimentan la duda sobre la independencia de sus sondeos de opinión. Algunas encuestas son distorsionadas intencionalmente y tienen defectos serios (por razones políticas o por otros motivos) y es necesario estar vigilantes para identificar las encuestas no científicas y llamar la atención sobre las normas de transparencia en el diseño de estos estudios. Un mecanismo para este tipo de esfuerzo es la Iniciativa para la Transparencia (IT) de Aapor. En una conferencia reciente en Ciudad de México, ${ }^{12}$ el presidente del Comité Coordinador, doctor Tim Johnson, enfatizó que esta iniciativa está ganando muchos adeptos entre quienes conducen encuestas en América Latina, aunque hay bastante espacio todavía para incrementar su lista de miembros.

Uno puede conjeturar qué otro factor que nutre el escepticismo público hacia las encuestas es un cambio cultural en el valor que se le atribuye a la investigación científica. En los Estados Unidos, el escepticismo que existe entre la opinión pública hacia la investigación científica en general parece ir de la mano con las crecientes dudas sobre las encuestas. Sin embargo, hay poca evidencia sobre dicho escepticismo hacia la investigación; en los Estados Unidos, el apoyo a la investigación cientifica ha permanecido constantemente alto desde 1985 hasta $2014 .^{13}$

Una tercera posibilidad es que los mismos éxitos alcanzados en el campo de la investigación de opinión pública con encuestas han avivado sin querer el escepticismo pú-

\footnotetext{
${ }^{12}$ La conferencia fue patrocinada por el CINE, el INE y Wapor.

${ }^{13}$ https://www.nsf.gov/statistics/2016/nsb20161/\#/data. Apéndice, tabla 7-19.
} 
blico. Por ejemplo, el desarrollo de nuevos algoritmos para identificar datos falsos en las encuestas ha mejorado nuestra capacidad de separar la paja del trigo, por así decirlo. La publicación de reportes sobre los éxitos detectando casos aislados de fraude en las encuestas ${ }^{14} 0$ en las ciencias sociales en general (por ejemplo, el caso reciente en los Estados Unidos de un estudiante de posgrado que falsificó las encuestas de su investigación ${ }^{15}$ ) alimenta el interés y la desconfianza en la calidad de este tipo de investigaciones. La aparición en los medios de noticias sobre cómo estos métodos han identificado unas cuantas manzanas podridas con frecuencia ensombrecen el gran número de encuestas que son validadas por los mismos análisis. ${ }^{16}$

Pero, además de la mayor transparencia, debería buscarse un aumento en nuestra capacidad de detectar problemas cuando las encuestas se encuentran aún en curso. Los algoritmos para detectar fraude nos permiten develar problemas en las encuestas después de concluido el trabajo de campo, lo cual es útil pero no ideal. En los últimos años han aparecido nuevas técnicas que pueden ayudar a detectar malas prácticas desde el momento mismo en que se está realizando el trabajo de campo, evitando que afecte al resto del proceso y la calidad de los datos. En este sentido, los últimos esfuerzos de Lapop se han concentrado en usar la tecnología para facilitar un cuidadoso monitoreo de las encuestas durante la realización del trabajo de campo. Este

\footnotetext{
${ }^{14}$ https://www.washingtonpost.com/news/monkey-cage/wp/2016/03/26/yesyou-can-trust-international-surveys-mostly/?utm_term=.a4dc69df524f.

${ }^{15} \mathrm{https}$ //fivethirtyeight.com/features/how-two-grad-students-uncovered-michaellacour-fraud-and-a-way-to-change-opinions-on-transgender-rights/

${ }^{16} \mathrm{El}$ creciente énfasis en el libre acceso a los datos, la transparencia y la replicación han tenido un efecto similar en todas las ciencias: algunos estudios no han sobrevivido al escrutinio. La consecuencia de las discusiones públicas sobre algunas retractaciones puede estar teniendo un efecto paralelo a la inquietud que algunos han manifestado en el terreno de la lucha contra la corrupción: al iniciar esfuerzos públicos por limpiar la casa, partes del público pueden ver la casa aún más sucia.

https://ecpr.eu/Filestore/PaperProposal/25a9a4d7-9618-44fb-898a-01f418d 8f265.pdf
} 
uso de tecnología ha transformado el entrenamiento de los entrevistadores, pasando de sesiones de formación y capacitación de un único evento a un proceso continuo y mucho más elaborado. Por ejemplo, al grabar pequeñas porciones de las entrevistas a través de los dispositivos móviles usados para recolectar los datos, los supervisores de campo pueden revisar y hacer observaciones sobre el desempeño de cada entrevistador a medida que el trabajo avanza. Ahora más que nunca, podemos estar seguros de que las preguntas son formuladas en el terreno de una forma que corresponda fielmente a cómo están escritas.

Desafortunadamente, los avances en el estudio científico de la metodología de encuestas no tienen lugar en un entorno estático que permita un crecimiento constante y creciente en la calidad de estas. Muchas mejoras van acompañadas, lamentablemente, de la aparición de nuevos problemas. Por ejemplo, a las dificultades ya mencionadas por otros participantes en este simposio me gustaria añadir y enfatizar los crecientes retos importantes a los que se enfrentan las entrevistas realizadas cara a cara debido al aumento de la delincuencia y la inseguridad en muchas partes de América Latina y el Caribe. La preocupación de ser victimizados lleva a muchas personas a no abrir la puerta y renunciar a participar en encuestas (este hecho podría estar relacionado en parte con lo señalado por algunas de las otras contribuciones a este simposio respecto del problema general de tener niveles de respuesta cada vez más bajos). Aún más, en áreas de alto riesgo, los entrevistadores tienen grandes incentivos para falsificar entrevistas y los equipos de trabajo tienen mayor dificultad para llegar a ciertos vecindarios para verificar las entrevistas. En algunos casos, pueden minimizarse los efectos que estos problemas pueden tener en la calidad de la encuesta usando nuevas tecnologías que permitan monitorear el trabajo de campo. Si bien existe la alternativa (discutida ya por otros colaboradores de este simposio) de poder hacer uso de encuestas con recogida de datos mul- 
timodales, lo cual permite a los entrevistadores contactar con comunidades que son inalcanzables personalmente a través del teléfono o por Internet. Sin embargo, queda mucho trabajo por hacer para refinar y validar estrategias que combinen distintas aproximaciones de recolección de datos en América Latina y el Caribe.

Una de las reticencias entre quienes dudan de las encuestas es que los sondeos de opinión pública y los pronósticos electorales han fracasado en sus predicciones respecto de una serie de consultas ciudadanas realizadas en 2016, como las convocatorias del referéndum de los acuerdos de paz en Colombia, el del brexit en el Reino Unido y las elecciones presidenciales en los Estados Unidos. Un énfasis exclusivo en estos errores corre el riesgo de pasar por alto las frecuentes predicciones acertadas ofrecidas por las encuestas electorales en los últimos tiempos (incluyendo, por ejemplo, el resultado del voto popular a favor de Hillary Clinton en las pasadas elecciones presidenciales en Estados Unidos). Pese a ello, es cierto que hay lecciones por aprender de dichos errores, como señalan también algunos de los colaboradores de este simposio. Precisamente dicho espiritu crítico ha llevado a los investigadores a discusiones públicas sobre cómo mejorar la precisión de las encuestas y los pronósticos electorales. Esto es importante. No obstante, con frecuencia en tales discusiones está implícita la sensación de que la investigación por muestreo es una ciencia exacta y que su valor sube o baja dependiendo de su éxito en el pronóstico de resultados particulares. Si no corregimos esta visión, nos convertiremos para siempre en don quijotes persiguiendo molinos de viento.

La investigación científica, y especialmente la que se lleva a cabo en las ciencias sociales, es probabilística. Quienes estudian el comportamiento político buscan identificar y entender patrones sistemáticos en la forma en la que las personas interactúan con la política. Las encuestas que indagan sobre las preferencias electorales de los ciudadanos nos permiten hacer esto. Por ejemplo, nos permiten apre- 
ciar que, en promedio, en América Latina, los ciudadanos de estos países son más dados a votar por los partidos de la izquierda política si son varones, pobres, no muy religiosos e indígenas y están a favor de la intervención del Estado en la economía, de promover políticas preventivas para enfrentar el crimen y de favorecer el matrimonio del mismo sexo y si desconfían más de los Estados Unidos (Carlin, Singer y Zechmeister, 2016). También en promedio, los latinoamericanos son más dados a votar contra el gobierno de turno cuando perciben que la economía nacional se ha deteriorado, creen que la corrupción es la norma entre los servidores públicos y se sienten inseguros en sus vecindarios (ídem). Necesitamos hacer más para resaltar ante la opinión pública el valor de este tipo de análisis de tendencias generales dentro de diferentes subgrupos de la población, más allá de cualquier intento de predecir el voto particular de cualquier individuo o grupo.

Las encuestas relevantes para entender las elecciones también pueden decirnos qué tan estables son las actitudes a través del tiempo. Por ejemplo, McCann y Lawson (2003) usaron un panel de encuestas realizadas a intervalos regulares durante los meses previos a la elección de 2000 en México para mostrar que, aunque el partidismo es más estable que otras actitudes potencialmente relevantes para las elecciones, tales como la autoubicación en el espectro de izquierda-derecha y las opiniones sobre políticas públicas, el partidismo también fluctúa a nivel individual durante la campaña. Dichas encuestas también pueden mostrarnos cómo patrones sobre actitudes relevantes para el voto se manifiestan de forma diferente en cada contexto. Por ejemplo, los análisis de datos de opinión pública nos muestran que el grado en el que los ciudadanos castigan electoralmente al gobierno de turno por la corrupción varía dependiendo del contexto económico: las personas que viven en áreas más prósperas son más tolerantes hacia la corrupción que aquellas que viven en zonas comparativamente más deprimidas (Zechmeister y Zizumbo-Colunga, 2013). 
Finalmente, proyectos de encuestas entre países repetidas a lo largo del tiempo -para América Latina y el Caribe, el Barómetro de las Américas y el Latinobarómetro- nos ayudan a interpretar el significado del comportamiento político en una perspectiva comparada. Por ejemplo, el Barómetro de las Américas Lapop de 2014 encontró que uno de cada cuatro brasileños estaba de acuerdo con que se podía confiar en alguna medida en las elecciones de su país. ¿Es ese porcentaje alto o bajo? Considerando los 18 países de América Latina incluidos en ese estudio, solo uno (Colombia) tenía un público que confiaba menos en las elecciones que Brasil. Aún más, las series temporales de esta misma encuesta comparada nos permiten apreciar, por ejemplo, que el respeto por las instituciones políticas de Brasil manifiesta una tendencia descendente (Russo, 2016). En resumen, con datos recogidos en el marco de proyectos de encuestas comparados repetidos en sucesivas series, puede apreciarse que ese $25 \%$ resulta bajo si se compara con otros países y si se observa su evaluación en el tiempo. Con posterioridad a la recogida de estos datos, las protestas políticas y la crisis crecieron en Brasil y la presidenta electa fue depuesta de su cargo para enfrentar un juicio ¿Los datos de esta encuesta predijeron el caos político? No, pero sugirieron la posibilidad de una gran inestabilidad política como consecuencia del creciente malestar ciudadano.

En sintesis, algunos ponen en duda el valor de las encuestas electorales. Estariamos en lo correcto al reconocer que hay muchos retos y oportunidades de mejora. No debe perderse de vista, sin embargo, que nuestra capacidad para producir encuestas de la más alta calidad ha crecido de forma extraordinaria en los últimos años con los nuevos métodos y tecnologías. La mayor disponibilidad de datos de opinión pública proporciona a un creciente número de analistas los medios con los cuales podemos mejorar nuestra comprensión de importantes regularidades empíricas en actitudes y comportamientos relevantes para las elecciones. Aún hay trabajo por hacer para continuar mejorando la pre- 
cisión científica y la validez de la investigación por encuestas en América Latina y en otros lugares. Aún queda más por hacer para educar al público general para que entienda este tipo de investigación y valore las encuestas cientificas y la transparencia en la investigación (incluyendo el reconocimiento de las limitaciones). También para que aprecie los análisis detallados de los datos de las encuestas que vayan más allá de predicciones particulares, en favor de contribuciones más comprehensivas que muestren cómo los ciudadanos evalúan sus sistemas políticos y participan en política.

\section{Referencias}

Asher, Herbert 2016 Polling and the Public: What Every Citizen Should Know. $9^{\text {th }}$ edition, Thousand Oaks, CA: CQ Press, Sage Publications.

Barómetro de las Américas por el Proyecto de Opinión Pública de América Latina (Lapop).

Carlin, Ryan E., Matthew M. Singer, and Elizabeth J. Zechmeister 2015 The Latin American Voter: Pursuing Representation and Accountability in Challenging Contexts. Ann Arbor, MI: University of Michigan Press.

McCann, James A., and Chappell Lawson 2003 "An Electorate Adrift? Public Opinion and the Quality of Democracy in Mexico" Latin American Research Review 38(3); 60-81.

Russo, Guilherme 2016 "Amid Brazil's Crises, Low and Declining Respect for Political Institutions" AmericasBarometer Topical Brief. (Abril 1). Nashville, TN: Lapop.

Zechmeister, Elizabeth J., and Mitchell A. Seligson 2012 "Public Opinion Research in Latin America" Chapter 31 in the Handbook on Latin American Politics, edited by Peter Kingstone and Deborah Yashar. NY: Routledge.

Zechmeister, Elizabeth J., and Daniel Zizumbo-Colunga 2013 "The Varying Political Toll of Concerns about Corruption in Good versus Bad Economic Times" Comparative Political Studies 46(10): 1190-1218. 